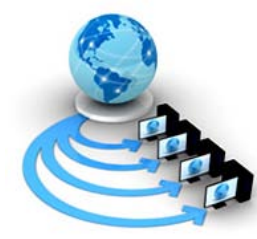

Volume 9, No. 2, March-April 2018

REVIEW ARTICLE

Available Online at www.ijarcs.info

\title{
SURVEY ON HOME SECURITY SURVEILLANCE SYSTEM BASED ON WI-FI CONNECTIVITY USING RASPBERRY PI AND IOT MODULE
}

\author{
Baswaraju Swathi \\ Sr. Assistant Professor, \\ Dept. of Information Science and Engineering, \\ New Horizon College of Engineering, \\ Bangalore, Karnataka, India
}

\author{
Somesh Balani \\ Students, Dept. of Information Science, \\ New Horizon College of Engineering, \\ Bangalore, Karnataka,India
}

\author{
Niza Barun Shrestha \\ Students, Dept. of Information Science, \\ New Horizon College of Engineering, \\ Bangalore, Karnataka,India
}

\begin{abstract}
This paper details the design and development of Wi-Fi connectivity based on home security surveillance camera using Raspberry Pi and an IoT Module reducing continuous human monitoring and controlling with enhanced performance and effective results.Adding wireless fidelity to embedded systems will open up various feasibilities such as worldwide monitoring and control, reliable data storage etc. This system comprises of wireless sensor nodes and a controller section for surveillance. Remote user alerts, live video streaming, and portability are the prime features of the system. Modular design is followed so that any number of nodes can be added or removed; data acquisition is used to sample signals and measure real world physical conditions. The user is warned via e-mails, phone calls or SMS after an unusual behavior occurs and an event notification is sent which shows the events captured by the camera. The IoT module eliminates the need for a microcontroller and wireless transceiver module in a sensor node, thus it makes the node compact, cost-effective and easy to use. The biggest advantage of the system is that the user can seek surveillance from anywhere in the world and can respond according to the situations.
\end{abstract}

Keywords: Raspberry Pi, Sensors, IoT, Embedded Systems, Microcontroller.

\section{INTRODUCTION}

In the $21^{\text {st }}$ century where the crimes regarding the invasion of someone else's property and robbing, stealing and much other antisocial behaviors has reached its peak, ensuring safety and security has an inevitable significance. And as, the impact of modern technology has escalated and advanced around the world, the need for security systems are been increasing constantly. Modern home needs intelligent systems with minimum human effort. With the advent of digital and wireless technologies, automated security systems become more intelligent. In the present situation, the requirements of security cameras in modern society become essential and effective for the home surveillance to prevent the house from any kind of danger.

Surveillance camera allows the users to have the remote view of his home from anywhere in the world and respond according to the situations. And, moreover, extra security features such as temperature sensors and motion detecting sensors can also be added for the better effectiveness of the system. Also, adding Wi-Fi to security systems enables faster data transmission and it will help the user to monitor and control the systems globally. Adding to all these features, the IoT module eliminates the necessity of a microcontroller or a computer and wireless transceiver module in a sensor node, thus it makes the node portable, compact, cost-effective and easy to use. Also, the creation of web servers helps the user to view the sensor status and the live video. This system also sends intruder's picture and the captured video to the owner by e-mail. The main aim of our project is to develop and implement a low cost, reliable, energy efficient, long range, globally accessible, storage effective surveillance system using Raspberry $\mathrm{Pi}$. The successful development of this Surveillance system [1] will allow all kinds of people to use it and prevent everyone to not to compromise their own and their family's safety and security.

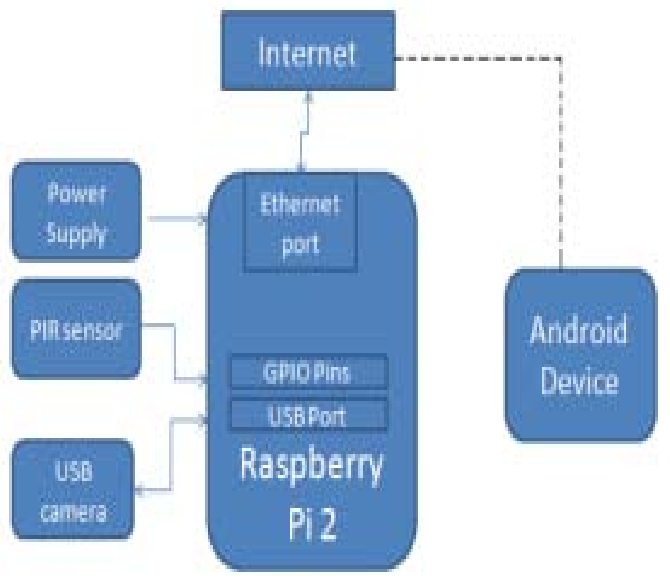

Fig1.Surveillance System

\section{LITERATURE SURVEY}

\section{A. An Internet of Things Approach for Motion Detection using Raspberry Pi}

The proposed system[2], would detect and take snapshots and videos of the motion when detected and upload to an 
external server. The major use of the 'Motion Detection' is at homes, buildings and also for surveillance for security for example of server rooms. This system simplifies the use of motion detection and is user-friendly which alerts the user by sending notifications when motion is detected. The motion sensor detects the change of activity in the video by analyzing it with a python script[1], if there is any difference in the video from the most recent frames, it would alert the user by snapshots and video recordings. The sensor settings can be customized to the user requirements. Real-time data processing and analytics which may arise incomplete datasets [4] in sensor settings should be considered along with clustering of datasets[5].

The project makes use of Raspberry Pi, a low priced credit card sized computer which is capable enough of working as a normal computer and uses a triple-layered architecture where the first layer is the motion detection layer which shows the outcome by the python script. The second layer produces the triggered actions as per the configuration file. And in the third layer, the triggered actions are executed where the user is notified about the activity through emails or pictures saved on the ftp/sftp server. The system is well tested and has given accurate results and notifications; it is an enhanced alternative system for expensive security system being used in the present day. The system is easy to install and doesn't require any other special modifications for implementing. The main advantage of the project is that the Raspberry $\mathrm{Pi}$ used, plays a 1080p resolution videos without lagging and is also used as a server for light traffic such as web traffic or DNS servers or NTP servers. The disadvantages found in the project are that the Raspberry Pi used cannot run X86 operating systems such as Windows and some Linux distributions and also applications, which require high $\mathrm{CPU}$ utilization.

\section{B. DESIGN AND IMPLEMENTATION OF THE MOBILE FIRE ALARM SYSTEM USING WIRELESS SENSOR NETWORKS}

This paper[6] proposes the design and implementation of mobile fire alarm system which uses wireless sensor networks; it is defined as a combination of large sensor nodes where each node can sense, process and communicate with its peers so as to work in an efficient manner. A sensor node that has a microprocessor has the ability of monitoring and wireless connectivity, the sensor shows its reading by a small screen. The sensors used in transmission such as temperature, humidity, pressure etc...moves the information on the wireless network to the data processing station where the reading is processed without the human intervention in the location of the physical environment. Sensors have limited energy stock components and the size of the memory is small, constant and changeful. The following modules are the sensor components:

1. Unit Transmission and reception

2. Power supply unit

3. Sensor Module

4. The unit of data storage and micro-controller for processing.

The readings obtained by the sensor nodes from the room are sent to the sink node, which is programmed to base station. Further readings are sent to the computer via the serial port. The programming was performed for the reading of sensor values.

//read value:

void loop(void)\{

uint_tchk=DHT11.read(DHT11PIN);

int sensorValue1=digitalRead(9);//fire

intsensorValue=analogRead(A1);//fire

int va2=analogRead(A0);//gas

int va2=digitalRead(8);//gas

//condition data:

if(va2 $==0$ )

\{client.println("GAS: No Gas");

if(va2==1)\{client.println("GAS: Has GAS”);

if(sensorValue1==0) \{client.println("FIRE No FIRE”);

if(sensorValue1==1) \{client.println("FIRE: Has FIRE”);

client.print(“Humidity: ”);

client.println(DHT11.humidity, DEC);

client.print(“Temperature(C):”);

client.println(DHT11.temperature, DEC);

The readings taken by the sensors depend upon the existence and absence of any human activity. The values are transmitted to the mobile device through the installed network connection. If the resultant values surpass the predefined threshold level, then the user is notified by a notification sent on their mobile phone to prevent a possible disaster. In case of any unusual activity occurs at home or office, a notification is sent to the user's personal mobile phone by the application on the phone. Thus, in this way, the paper proposes a system that ensures the user's safety and helps to keep track of their home or office's safety within the limits of the presence of the router connected between mobile phones and devices. It provides a low-cost for industrial and home safety systems. The system proposed in the paper has a very simple design, which corresponds simple hardware circuits that permits each user to use this wireless home safety system. The only disadvantage found is that it is easily troubled by surroundings (walls, microwave, large distances due to signal attenuation, etc.).

\section{DESIGN AND IMPLEMENTATION OF AN EMBEDDED HOME SURVEILLANCE SYSTEM BY USE OF MULTIPLE ULTRASONIC SENSORS}

The proposed paper[7] puts forth a design and implementation of a home surveillance system using on an embedded system along with multiple ultrasonic sensor modules, which helps the system to perform efficiently as well as makes the system reliable. The ultrasonic transmission will spread at a beam angle and for that multiple ultrasonic receiver are used to receive the ultrasonic transmission The system will sense the intruder activity as when an intruder passes through the sensing area, the ultrasonic transmission will be blocked by human body and receivers won't receive any transmission from the ultrasonic transmitter.

To show the system's reliability, Majority Voting Mechanism (MVM) is used for a group of sensors. If more than half of the sensors in a group sense a signal blocking, then the majority voting mechanism program starts the Web 
camera. The mathematical equation and the sensing experiment show the improvement in system's reliability. According to our MVM, the resolution count must be greater than $0.5 \mathrm{x} \mathrm{n}$, with $\mathrm{n}$ being the total number of sensors. To fit the extreme value of $n$ we use $w x n$ to deduce the relationship between $P_{\text {multiple }}(n)=P_{m}$ and $P_{\text {single }}=P_{s}$ in the extreme value of $n$.

$$
P_{m}=\left(P_{s}\right)^{w \cdot n} \cdot\left(1-P_{s}\right)^{(1-w) \cdot n} \cdot \sum_{r=0}^{(1-w) \cdot n}\left[\left(\begin{array}{c}
n \\
r+w \cdot n
\end{array}\right) \cdot\left(\frac{P_{s}}{1-P_{s}}\right)^{r}\right]
$$

(1)

We define

$$
f(k)=\left(\begin{array}{c}
n \\
k+w \cdot n
\end{array}\right) \cdot\left(\frac{P_{s}}{1-P_{s}}\right)^{k}
$$

And,

$$
k=\{0,1,2,3, \ldots,[(1-w) \cdot n-1],[(1-w) \cdot n]\}
$$

As we expect that $\sum_{k=0}^{(1-w) \cdot n} f(k)$ determine whether $\mathrm{f}(\mathrm{k})$ is a decreasing function. From the ratio test for the convergence function, we learn that increasing the $n$ value decreases the ratio gradually for each $\mathrm{f}(\mathrm{k})$. The relationship is as follows.

$$
\begin{aligned}
& 1>\left|\frac{f(1)}{f(0)}\right|>\left|\frac{f(2)}{f(1)}\right|>\left|\frac{f(3)}{f(2)}\right|>\cdots>\left|\frac{f[(1-w) \cdot n-1]}{f[(1-w) \cdot n-2]}\right|>\left|\frac{f[(1-w) \cdot n]}{f[(1-w) \cdot n-1]}\right| \\
& \text { Let } n \rightarrow \infty, \text { so } \\
& \lim _{n \rightarrow \infty}\left|\frac{f(1)}{f(0)}\right|=\left(\frac{1-w}{w}\right) \cdot\left(\frac{P_{s}}{1-P_{s}}\right)<1
\end{aligned}
$$$$
\text { We let } 1 / 2 \leq w<1 \text { and } P_{s}>w
$$$$
P_{m}=\left.\left(P_{s}\right)^{w n n} \cdot\left(1-P_{s}\right)^{(1-w) \cdot n} \cdot \sum_{r=0}^{(1-w) n} f(k) \Rightarrow \lim _{n \rightarrow \infty}\left(P_{m}\right)\right|_{\left(P_{s}>w\right)}=1
$$

If we let $\overline{P_{m}}$ represent the miss rate of sensors, we learn that $\overline{P_{m}}=1-P_{m}$ and $\overline{P_{m}}=0$ in this case.

We rewrite (1), by letting $P_{s}<w$ and thus deducing $\overline{P_{m}}=1-P_{m}$ by the ratio test.

$$
\begin{aligned}
& \overline{P_{m}}=\left(P_{s}\right)^{(1-w) \cdot n} \cdot\left(1-P_{s}\right)^{w \cdot n} \cdot \sum_{r=0}^{(1-w) \cdot n} g(k) \\
& \left.\Rightarrow \lim _{n \rightarrow \infty}\left(\overline{P_{m}}\right)\right|_{\left(P_{s}<w\right)}=1
\end{aligned}
$$

According to (2) and (3), the sensing probability of multiple sensors must be greater than the sensing probability of any single sensor. We know that when $\mathrm{P}_{\text {single }}$ is greater than 0.5 , the $\mathrm{P}_{\text {multiple }}(\mathrm{n})$ will be greater than 0.5 .

The sensing probability is improved by the use of multiple sensors having an MVM. However, an increment in the cost is resulted due to the use of multiple sensors, the voting circuit, and the amplifier circuits. In spite of that, the occurrences of false alarms are reduced due to the proposed home surveillance system's reliability. The plus point of the proposed system is that it can be used in detecting highfrequency ultrasonic transmission. The disadvantage is that system may operate with less speed as expected.

\section{DESIGN AND IMPLEMENTATION OF A HOME EMBEDDED SURVEILLANCE SYSTEM WITH ULTRA-LOW ALERT POWER}

This paper[8] proposes a design of a home embedded surveillance system with ultra-low-power. Here, Pyroelectric Infrared Sensors (PIR) and pressure sensors have been built around a microcontroller with ultra-low alert power as the alert group in windows and doors where an intruder must pass through. In this, the PIR sensor generates a signal upon any kind of activity and sends it to the system which senses the signal. When the sensor detects the presence of any unauthorized person, it directly triggers the alarm and a call alert is sent to a predefined number through a GSM modem. The low- power alert sensors wake up the MCU (Micro Controller Unit) which has power management for the ultrasonic sensors and PIR sensors indoors. Also, in the design introduces, Majority Voting Mechanism (MVM) is used to manage sensor groups to enhance the probability of multiple sensors sensing. The embedded system reads the sensor signal sent by MCU, after which the program triggers the Web camera.

The working of the system was analyzed and it shows that the system reduces the memory consumption and also decreases the power consumed by the system.

Making use of MVM improves the sensing reliability of the sensors placed indoor. The readings of the sensors are directly sent to the embedded system which then decides to whether trigger the web camera or to images and upload those images to the web server via the internet. Another feature of the system reduces the power consumption and prevents wastage of energy by putting the MCU in sleep mode when there is no activity.

The following table shows the sensors used in different operating states:

Table 1

\begin{tabular}{|l|l|l|}
\hline System State & Alert State & Detection State \\
\hline PIR Sensors & Use & Use \\
\hline Pressure Sensor & Use & Not in Use \\
\hline Ultrasonic Sensor & Not in Use & Use \\
\hline
\end{tabular}

The following table shows the power consumption of $\mathrm{s}$ Single Sensor Group:

Table 2

\begin{tabular}{|l|l|l|}
\hline System State & $\begin{array}{l}\text { Alert State } \\
\text { Power } \\
\text { Consumption } \\
\mathrm{mW}\end{array}$ & $\begin{array}{l}\text { Detection State } \\
\text { Power } \\
\text { consumption } \\
\text { Mw }\end{array}$ \\
\hline PIR sensors & 1.05 & 7.35 \\
\hline Pressure sensor & 0.8 & 1.5 \\
\hline $\begin{array}{l}\text { Ultrasonic } \\
\text { Sensor }\end{array}$ & Not in use & 256.08 \\
\hline
\end{tabular}

The above two tables are obtained results after the design proposed in the paper was developed and implemented. In this design, multiple sensor groups with low power consumption are used for the detection of an intruder. The MCU stays in a sleep state, unlike the traditional surveillance system which stays in the detection state. They reduce the power consumption in the alert or sleep state by 
10.9 times by remaining $90 \%$ in the alert state and $10 \%$ in the detection state, and also, two sensor groups to improve the detection reliability of the alert state has been used. In addition, the home embedded surveillance system reduces unnecessary memory consumption for the capture of images without an intruder, compared to previous surveillance systems. The low power consumption and the reduction in the unnecessary memory consumption can be considered as the plus point of the proposed system but the system is examined to be not reliable in various situations

\section{E. WIRELESS SENSOR NETWORKS FOR HABITAT MONITORING}

In this paper[9], a thorough study of using wireless sensor network to real-world habitat monitoring is given. A specific habitat monitoring application that generally represents the domain is developed and described by the paper. An architecture of the system is put forth to address the developed requirements and, an instance of the architecture for monitoring seabird nesting environment and behavior is presented. The network developed recently is said to consist of 32 nodes on a small island off the coast of Maine streaming useful live data onto the web. The application- driven design proposed in the paper aids to identify essential areas of further work such as health monitoring, network retasking, data sampling and communications.

A tiered architecture is designed and developed. The sensor nodes that carry out general purpose computing and networking and also, application-specific sensing are integrated into the bottom-most level of the architecture. The sensor nodes send their data to the sensor network gateway through the sensor network.

At last, the resultant data is showed to the scientists through a user interface. Gizmo, commonly known as mobile phones, can be used to interact with any of the networks whether it is a field of across the world connected to the database replica. With the aid of the various sensors that are being used, the system that is proposed by the paper is expected to provide good power management with the cost being a little high.

The characteristics of the various sensors that are being used in the proposed system are illustrated by the table shown below:

Table 3

\begin{tabular}{|l|l|l|l|l|l|}
\hline \multicolumn{1}{|c|}{ Sensor } & \multicolumn{1}{c|}{ Accuracy } & Interchangeability & \multicolumn{1}{c|}{ Sample Rate } & \multicolumn{1}{c|}{ Startup } & \multicolumn{1}{c|}{ Current } \\
\hline Photo resistor & N/A & $10 \%$ & $2001 \mathrm{~Hz}$ & $11 \mathrm{~ms}$ & $0.245 \mathrm{Ma}$ \\
\hline $\mathrm{I}^{2} \mathrm{C}$ Temperature & $1 \mathrm{~K}$ & $0.21 \mathrm{~K}$ & $2.01 \mathrm{~Hz}$ & $501 \mathrm{~ms}$ & $0.010 \mathrm{Ma}$ \\
\hline $\begin{array}{l}\text { Barometric } \\
\text { Pressure }\end{array}$ & $1.6 \mathrm{mbar}$ & $0.6 \%$ & $10 \mathrm{~Hz}$ & $500 \mathrm{~ms}$ & $0.010 \mathrm{Ma}$ \\
\hline $\begin{array}{l}\text { Barometric } \\
\text { Pressure Temp }\end{array}$ & $0.8 \mathrm{~K}$ & $0.25 \mathrm{~K}$ & $10 \mathrm{~Hz}$ & $500 \mathrm{~ms}$ & $0.778 \mathrm{Ma}$ \\
\hline Humidity & $2 \%$ & $3 \%$ & $500 \mathrm{~Hz}$ & $500-3000 \mathrm{~ms}$ & $0.190 \mathrm{Ma}$ \\
\hline Thermopile & $3 \mathrm{~K}$ & $5 \%$ & $2000 \mathrm{~Hz}$ & $200 \mathrm{~ms}$ & $0.136 \mathrm{Ma}$ \\
\hline Thermistor & $5 \mathrm{~K}$ & $10 \%$ & $2000 \mathrm{~Hz}$ & $10 \mathrm{~ms}$ & \\
\hline
\end{tabular}

\section{F. IOT BASED SMART SURVEILLANCE SECURITY SYSTEM USING RASPBERRY PI}

This paper[10] proposes a detailed design and implementation of an IoT based smart surveillance security system using Raspberry Pi. The home surveillance security system is one of the most crucial components of a luxurious home. The smart home technology provides the added security as well as energy-saving technique to the consumers. Nowadays, everyone wants a secure and comfortable life. The system uses a web camera which records images when an intruder enters a room; the web camera detects the motion and captures it. The user is notified of the intruder activity via email or SMS. The email with the attachment of intruder's image and a text message with a warning will be sent to the user. The monitoring system is connected and implemented to the server. Use of Raspberry $\mathrm{Pi}$, which is a credit card sized computer perfectly capable of working as a normal computer does, provides high video resolution without any lag. The Raspberry Pi runs Linux form operating systems; and is a significant extra powerful device but that power arises with some responsibilities. An algorithm for motion detection is given below:

\section{Algorithm for Motion Detection:}

1. Process motion detection.

2. Calculation of the mean of a particular shade in that first frame.

3. Waiting for X sec.

4. Calculation of the mean of a particular shade in that second frame.

5. Condition absolute (avgFrame1-avgFrame2) > threshold then.

6. Motion detected.

Motion Detection algorithm can’t examine a spinning thing - a thing i.e. changes.

The monitoring system contains hardware as well as the software operations. The hardware implementation carried out the Raspberry $\mathrm{Pi}$, and the software implementation carried out the software program that was programmed in the Raspberry $\mathrm{Pi}$ and the concern Person's computer. Raspberry Pi is the central core part of the whole proposed system. It takes the command from either an Android tablet or PC and according to the command, controls the appliances connected to it on the output port. It is connected to an Android tablet or phone via a router by using Wi-Fi or Ethernet cable. It is also used as a web server to store the 
status of appliances in the database and give it to the PHP web application to display on the webpage.

The system can be further improvised by adding more sensors like the infrared emitting system to detect the people face in case of any burglary where they wear masks or cover their face. Sensors for gas, fire, and smoke would be another special feature of the system to prevent accidents or disasters. The Raspberry Pi used, produces high-resolution videos without any lag. But, the major drawback of the proposed system is that the motion detection can't catch any moving or spin things. Also, faces of a person that is covered using a mask or anything cannot be captured.

\section{G. SECURITY SYSTEM USING RASPBERRY PI}

In this paper[11], an intelligent surveillance system is proposed that continuously monitors the targeted area and detects the motion in each and every frame. If the system detects motion in the targeted area then a notification is automatically sent to the user by SMS and the video starts getting recorded till the motion is stopped. On implementing the method illustrated in the paper, the required memory space for storing the video is also reduced since it doesn't store the entire video but stores the video when a motion is detected. OpenCV(computer vision/ machine vision) technology and raspberry pi are used to achieve real-time video processing. Moreover, the disadvantage of using locker systems for the protection of jewelry and money is also mention and how the current security systems are open to attack. Therefore, to overcome this disadvantage an embedded security system is used commonly known as, Raspberry Pi operated by OpenCV, which also uses a low power supply. If there is an intruder in the surveillance area the Raspberry Pi sends alert information to the authority by mail along with the intruder image which is obtained by the video that captures the image continuously.

Motion detection is implemented by using a differential comparison method which works on the principle of image comparison. Three frames are taken from the video capturing unit which are continuous frames used for the detection. To detect the motion, the difference of the 3 frames taken simultaneously is compared. If there is a change then between the values, it means that a motion is detected. If there is no change then it means that there is no motion in the captured frames, after which it will again start capturing the video and redo the same procedure in an iterative manner.

The proposed system also consists of a GSM modem which is connected to the system by means of a USB port present in the Raspberry Pi. The USB port acts as an alert system, which immediately calls the SMS function, once the motion is detected. GSM modem contains all the authentication details and the list of phone numbers to which the alert message needs to be sent. Open computer vision is a tool that allows the webcam surveillance through Raspberry Pi. It is a library function that enables all real-time video and image processing i.e., enabling the computer to see. All the real-time video processing systems were operated with the help of this library. The programming languages such as $\mathrm{C}$, $\mathrm{C}++$, Python, and Java have been used for real-time processing. The python scripting has been used since the hardware plot is Raspberry Pi which will provide the better support for python scripting.

Furthermore, it does not need any manpower to monitor the entire video continuously and thus, manpower is also reduced. The author of the paper aims to use the proposed system in all the secured places such as bank lockers, ATM centers, etc. The main advantages of the proposed system is that it provides a safe security system over a highly arcane area at a low cost; the system is smaller, lighter with lower power consumption; and it also reduces the video storage system to a huge extent since it will store the video only if it detects any motion and also, the cost of the entire system is also reduced.

\section{FUTURE WORK}

This paper aims at providing an efficient surveillance camera system for home security. Future work concerns deeper analysis on the existing surveillance system and a detailed improvisation and modification based on the analysis. We plan to use a motion detector sensor with the PIR sensors for any intruder detection and for detecting the fire in the environment, temperature sensors are proposed to be used. Furthermore, face recognition was found to be a major drawback for the present systems, so, to overcome this disadvantage we intend to use a face recognition mechanism so that the system does not confuse the owner as an intruder. In addition to all these, we also propose to build an android application that sends a notification to the user if any unusual activity is noticed in the environment so that the whole agenda of the system is accomplished.

\section{CONCLUSION}

In this paper, we introduce an active surveillance system where the user will get alerts when an event occurs through Phone calls or SMS, emails, etc. Intruder and Fire detection are the most important features of the system. The system is developed using RaspberryPi and IoT module to provide real-time surveillance. The system will send intruder's captured images and video to the owner via email. The sensor status and live video can be streamed using the Web server's facility. The system is developed with an aim to provide security of everyone's home with less cost and userfriendly for common people to be able to afford it.

\section{REFERENCES}

[1] Priya B Patel, Viraj M Choksi, SwapnaJadhav and M B Potdar. "Article: Smart Motion Detection System using Raspberry Pi.” International Journal of Applied Information Systems 10(5):37-40, February 2016.

[2]. AamirNizam Ansari, Mohamed Sedky, Neelam Sharma and Anurag Tyagi, "An Internet of Things Approach for Motion Detection using Raspberry Pi” International Conference on Intelligent Computing and Internet of Things (IC1T), 2015, IEEE.

[3] Priya B Patel, Viraj M Choksi, SwapnaJadhav and M B Potdar. "Article: Smart Motion Detection System using Raspberry Pi.” International Journal of Applied Information Systems 10(5):37-40, February 2016.

[4]BaswarajuSwathi, Sonia Singh, Sujithraks, "INCLUSIVE ANALYSIS OF INCOMPLETE DATA SETS USING IkNNSEARCH”, International Journal of Innovative Research in Computer and Communication Engineering, Vol.5, Special Issue 5, June 2017. 
[5] Subiksha, Pallavi, B.Monica,"Clustering of Datasets Using KMeans Algorithm in SPARK”, IJIRCCE, vol5, issue 5, May 2017.

[6]. Muheden, Karwan, EbubekirErdem, and SercanVanin, "Design and implementation of the mobile fire alarm system using wireless sensor networks", IEEE Int.Symp.Computational Intelligence and Informatics, 2016, pp. 000243-000246.

[7]. Bai, Ying-Wen, Li-SihShen, and Zong-Han Li, "Design and implementation of an embedded home surveillance system by use of multiple ultrasonic sensors”, IEEE Transactions on Consumer Electronics 56, no. 1 (2010).

[8] .Bai, Ying-Wen, Zi-Li Xie, and Zong-Han Li, "Design and implementation of a home embedded surveillance system with ultra-low alert power”, IEEE Transactions on Consumer Electronics 57, no. 1 (2011)
[9]. Alan Mainwaring, Joseph Polastre, Robert Szewczyk, David Culler, John Anderson,”Wireless Sensor Networks for Habitat Monitoring.” WSNA’02, September 28, 2002, Atlanta, Georgia, USA.

[10]. Neha Patil, ShrikantAmbatkar, and SandeepKakde,”IoT Based Smart Surveillance Security System using Raspberry Pi", International Conference on Communication and Signal Processing, IEEE, April 6-8, 2017.

[11]. ShakthiMurugan.K.H, V.Jacintha,

S.AgnesShifani,"Security System using Raspberry PI" Third International Conference on Science Technology Engineering \& Management (ICONSTEM), IEEE,2017. 